\title{
PREVALENCE OF REFLUX OESPHAGITIS IN EGYPTIAN PATIENTS WITH LIVER CIRRHOSIS
}

\author{
By \\ ABDOU MABROUK EL-SHAFEI ${ }^{*}$, DIAA MOHAMMAD EL-TIBY ${ }^{1}$, \\ SHERIF ALI ABDELAZIZ ${ }^{1}$, AHMED ATEF ABDEL-FATAH ${ }^{1 *}$, \\ and ABDULLAH MUSTAFA GAAFAR ${ }^{2}$
}

Departments of Tropical Medicine ${ }^{1}$ and Clinical Pathology ${ }^{2}$, Faculty of Medicine, Al-Azhar University, Cairo, Egypt ( ${ }^{*}$ Correspondence:drahmedatef87@gmail.com)

\section{Abstract}

Reflux esophagitis (RE) is increasing in prevalence in Egypt. There are few studies on the prevalence and factors related to RE in patients with liver cirrhosis. This study determined the prevalence of RE in patients with liver cirrhosis and the possible related predictors of RE. This study was conducted on 150 patients with liver cirrhosis who underwent upper GI endoscopy for different indications. GERD, if present was classified according to Los Angeles Classification, Oseophageal biopsies taken to exclude Barret's oesophagus and microscopic reflux oesphagitis.

The results showed that 36 patients had GERD (24\%). The most prevalent was GERD grade (B). Symptomatic GERD was highly prevalent in patients with liver cirrhosis, reported in about $(48.67 \%)$. Heart burn was the chief symptom with a significant relationship between GERD and severity of the liver disease as assessed by Child-Turcotte-Pugh scoring system. A significant relationship between the severity of GERD and the degree of ascites was demonstrated, as GERD grade (C) was present more frequently in patients with marked ascites. The presence of GERD was significantly associated with the esophageal varices, which could be amechanical factor contributing to esophageal dysmotility and predisposing to GERD, patients with no GERD by endoscopy (114) microscopically showing microscopic oesphagitis (29.8\%), RE (36.8\%), while patients with GERD by endoscopy(36) microscopically showing barrets oesphagus (22.3\%), RE with mild activity (41.6\%), RE with moderate activity (36.1\%).

Key words: Egypt, Patients, Reflux oesphagitis, Liver cirrhosis

\section{Introduction}

Gastroesophageal reflux disease (GERD), a highly prevalent disorder, is defined as chronic disorder resulting from the retrograde flow of gastroduodenal contents into the esophagus or adjacent organs, and producing a variable spectrum of symptoms, with or without tissue damage. Transient inappropriate relaxation of lower esophageal sphincter (LES) is the predominant pathophysiologic mechanism in the majority of GERD patients. Gastroparesis and a reduced LES pressure play a significant role in patients with moderate to severe disease, only one half of GERD patients present with esophageal erosions namely reflux oesphagitis (Fock et al, 2009). Symptoms of GERD typically include dyspepsia, pyrosis, or tissue da-mage outside esophagus, such as the pharynx, larynx, and trachea (Hammer et al, 2009). GERD was common condition affecting $25-40 \%$ of the population worldwide with impact on patient quality of life (Cooper et al, 2008). GERD is the most common and costly digestive disease. Annual direct costs for managing GERD are estimated to exceed billion dollars (Lemme et al, 2009). Symptomatic RE impairs quality of life of patients with CLD, so treatment of symptomatic RE should considered in order to improve quality of life in patients with CLD (Suzuki et al, 2008).

GERD was considered in patients with CLD, especially patients with portal hypertension and liver cirrhosis, have clinical manifestations, such as esophageal varices, ascites, and edema. Some studies have been conducted regarding the role of esophageal varices in the development of esophageal motor disorders and abnormal gastroesophageal reflux in these patients (Suzuki et al, 2008). Ascites could be a factor promoting gastroesophageal reflux and it has been questioned whether or not reflux would fa- 
vor the rupture of varices. However, there are few studies on the prevalence of RE and factors related to RE in patients with CLD (Navarro et al, 2008).

$\mathrm{RE}$ in cirrhotic patient with oesophageal varices were well known and great importance was paid to oesophageal dyspepsia as risk factor for the rupture and bleeding of ovs as in cirrhotic patient increased contact time between pepsin and ovs could lead to mucosa eventual erosion and ovs bleeding (Garcia-Tsao et al, 2008; Okamoto et al, 2008).

\section{Subjects and Methods}

Prospective study was carried out on 150 Egyptian cirrhotic patients with no past history of haematemsis who submitted to endoscopy from the inpatient and outpatient clinics of tropical medicine Al Azhar University Hospitals. The study was approved by the local ethics committee, Faculty of Medicine, Al-Azhar University. The excluded patients were those with systemic disease related to esophageal motor disorders and/or gastroesophageal RE (progressive systemic sclerosis, diabetes mellitus, and neuromuscular, chronic users of drugs that influence esophageal motility (calcium channel blockers, theophylline \& nitrate), platelet cou-nt $<150,000 / \mathrm{mm}$ and INR $>1.2$

All patients were subjected to thorough history taking and clinical examination, laboratory tests including CBC, serum ALT, AST, albumin, bilirubin, prothrombin time and INR, renal function test. Evaluation of liver cirrhosis severity was obtained in each cirrhotic patient with Child-Turcotte Pugh score. The system relied on clinical and laboratory evaluation including ascites, encephalopathy grade, serum albumin, bilirubin and prothrombin time (Christensen et al, 1984), as well as abdominal ultrasound assessed liver texture, splenic size and amount of ascites.

Technique (upper endoscopy): Patients came to endoscopy unit after an overnight fasting. Each patient was given intravenous sedation with midazolam, in a titrated dose of up to $0.1 \mathrm{mg} / \mathrm{kg}$ before the endoscopy. Care- ful procedure explanation was done to the patient, including risks and benefits, with informed gave written consent for the procedure.

I- GERD, if present was classified according to Los Angeles Classification (Lundell et $a l$, 2009): I- LA grade A: One (or more) mucosal break no longer than $5 \mathrm{~mm}$, not extend bet- ween the tops of two mucosal folds. II-

II-LA grade A: One (or more) mucosal break no longer than $5 \mathrm{~mm}$, that does not extend between the tops of two mucosal folds. III- LA grade B: One (or more) mucosal break more than $5 \mathrm{~mm}$, which does not extend between the tops of two mucosal folds.

IV-LA grade C: One (or more) mucosal break that is continuous between the tops of two or more mucosal folds, but which involves less than $75 \%$ of the esophageal circumference.

V-LA grade D: One (or more) mucosal break but which involves at least $75 \%$ of the oesophageal circumference.

Esophageal varices (EV) were classified according to Maratka classification (1989) according to the degree of protrusion into the lumen when the esophagus is maximally relaxed and inflated with air: Grade I: Varices were hardly noticeable protrusion, Grade II: Varices were protruded up to $1 / 4$ of the lumen, Grade III: Varices were protruded up to $1 / 2$ of the lumen, and Grade IV: Varices were protruded greater than $1 / 2$ of the lumen.

Esophageal biopsies: Esophageal biopsies were taken for histopathological study; biopsy was preserved in $10 \%$ formalin till needed.

Statistical methods: SPSS statistical software package (V. 17.0, Echo soft Corp., USA, 2008) was used for data analysis. Results were expressed as means \pm standard deviation of the means (SD). Differences between groups were analyzed either by using the Chi square test or student's t-test and nonparametric (Mann Whitney test) for comparison between two groups or ANOVA 
test for multiple group comparison. Spearman rank correlation coefficient was used to determine significant correlations among different parameters. Analysis was done using Statistical Analysis System, version 6.03, on an IBM at personal computer: SD: standard deviation, $\mathrm{M}$ : mean, and P-value.

\section{Results}

A total of 150 patients with liver cirrhosis underwent upper GI endoscopy for different indications. They were 104 males \& 46 females $(69.3 \% \& 30.67 \%$ respectively), with ages from 30 to 78 years $(50.50 \pm 10.94)$. 22 $(61 \%)$ patients with GERD were above 60 years. GERD was endoscopically evident in
36 patients (24\%), 30 patients $(80.5 \%)$ were males and 6 patients were females $(19.5 \%)$, GERD grade B was the most prevalent entity $(44.4 \%)$. Heartburn was the most presenting complaint in all symptomatic patients (73 patients i.e. $48.67 \%$ ), followed by epigastric pain in 58 patients $(38.67 \%)$, while acid regurgitation was in $41(27.33 \%)$ patients. Heart burn was presented in all patients with GERD grade (C), but, without significant relationship between various reflux symptoms and GERD different degrees.

Details are given in tables $(1,2,3,4,5$ \& $6)$.

Table 1: Base line clinical symptoms of groups

\begin{tabular}{|l|c|}
\hline Characteristics & Liver cirrhosis group $(\mathrm{n}=150)$ \\
\hline Typical symptoms & $73(48.67 \%)$ \\
\hline Heart burn & $73(48.67 \%)$ \\
\hline acid regurgitation & $41(27.33 \%)$ \\
\hline Excessive salivation & $9(6 \%)$ \\
\hline Epigastric pain & $58(38.67 \%)$ \\
\hline Atypical symptoms & $8(5.33 \%)$ \\
\hline Throat clearing & $8(5.33 \%)$ \\
\hline Hoarsness of voice & $1(0.67 \%)$ \\
\hline Chronic cough & $2(1.33 \%)$ \\
\hline Nocturnal asthma & $0(0 \%)$ \\
\hline
\end{tabular}

Heart burn was seen in all patients with

between various symptoms of reflux and GERD grade (C), but without significance GERD different degrees $(\mathrm{P}>0.05)$.

Table 2: Typical and atypical symptoms in relation to different GERD grades.

\begin{tabular}{|l|c|c|c|c|}
\hline Characteristics & GERD $(\mathrm{A})(\mathrm{n}=13)$ & GERD $(\mathrm{B})(\mathrm{n}=16)$ & GERD $(\mathrm{C})(\mathrm{n}=7)$ & $\mathrm{P}$ value \\
\hline Heart burn $(\mathrm{n}=24)$ & $8(60 \%)$ & $9(55.6 \%)$ & $7(100 \%)$ & $0.073(\mathrm{NS})$ \\
\hline Acid regurgitation $(\mathrm{n}=14)$ & $5(40 \%)$ & $5(27.8 \%)$ & $4(62.5 \%)$ & $0.245(\mathrm{NS})$ \\
\hline Excessive salivation $(\mathrm{n}=4)$ & $3(23 \%)$ & $1(6.25 \%)$ & $0(0 \%)$ & $0.222(\mathrm{NS})$ \\
\hline Throat clearing $(\mathrm{n}=3)$ & $1(6.7 \%)$ & $0(0 \%)$ & $2(28.6 \%)$ & $0.077(\mathrm{NS})$ \\
\hline Hoarsness of voice $(\mathrm{n}=0)$ & $0(0 \%)$ & $0(0 \%)$ & $0(0 \%)$ & --- \\
\hline Chronic cough $(\mathrm{n}=0)$ & $0(0 \%)$ & $0(0 \%)$ & $1(14.3 \%)$ & $0.121(\mathrm{NS})$ \\
\hline Nocturnal asthma $(\mathrm{n}=0)$ & $0(0 \%)$ & $0(0 \%)$ & $0(0 \%)$ & --- \\
\hline
\end{tabular}

Values expressed as number $(\%) . N S=$ Not significant $=p>0.05$.

Ascites was in 30 patients with GERD. had marked ascites with highly significant Majority of GERD grade A patients were presented with tense ascites (38.46\%), half of them with GERD grade B had moderate ascites, while $85.7 \%$ with GERD grade C data concerning the relation between the different degrees of ascites and different grades of GERD (P<0.001).

Table 3: Association between different grades of GERD and degree of ascites in groups

\begin{tabular}{|l|c|c|c|c|}
\hline Characteristics of ascites & GERD $(\mathrm{A})(\mathrm{n}=13)$ & GERD $(\mathrm{B})(\mathrm{n}=16)$ & GERD $(\mathrm{C})(\mathrm{n}=7)$ & P value \\
\hline Mild $(\mathrm{n}=35)$ & $3(23.01 \%)$ & $1(6.25 \%)$ & $0(0 \%)$ & \\
\cline { 1 - 4 } Moderate $(\mathrm{n}=41)$ & $4(30.76 \%)$ & $9(56.25 \%)$ & $1(14.3 \%)$ & \\
\cline { 1 - 4 } Marked $(\mathrm{n}=31)$ & $5(38.46 \%)$ & $1(6.25 \%)$ & $6(85.7 \%)$ & \\
No $(\mathrm{n}=43)$ & $1(7.69 \%)$ & $5(31.25 \%)$ & $0(0 \%)$ & \\
\hline
\end{tabular}

Values expressed as number $(\%)$.**p $<0.001=$ highly significant

In studied group, 33 patients $(22 \%)$ were classified Child A, 35 patients (23.3\%) were classified Child B, 82 patients $(54.7 \%)$ were classified Child $\mathrm{C}$, showing statistically 
highly significant difference between GERD and modified Child score (severity of liver damage), $p<0.001$. Majority of patients of
GERD grade A, B \& $\mathrm{C}$ were categorized as Child C $(53.8 \%, 56.2 \%$ \& $85.7 \%$ respectively), but without significant $(\mathrm{P}>0.05)$.

Table 4: Child-Turcotte-Pugh scoring system to assess severity of liver cirrhosis in relation to GERD

\begin{tabular}{|l|c|c|}
\hline Characteristics & GERD $(\mathrm{n}=36)$ & \multirow{2}{*}{ P value } \\
\hline Child A $(\mathrm{n}=33)$ & $6(18.1 \%)$ & 0.05 \\
\hline Child B (n=35) & $8(23 \%)$ & \\
\hline Child C (n=82) & $22(27 \%)$ \\
\hline \multicolumn{2}{|c|}{ Values expressed as number $(\%) \cdot \mathrm{p}<0.05=$ significant. }
\end{tabular}

A total of 32 patients $(88.89 \%)$ with GERD had different grades of esophageal varices, others $(11.11 \%)$ showed no significant difference $(\mathrm{P}=0.011) .53$ patients had recent variceal bleeding, 32 of them $(60 \%)$ had GERD with a highly significant difference between GERD and signs of recent bleeding.

Table 5: Association between GERD and presence of esophageal varices

\begin{tabular}{|c|c|c|}
\hline Presence of varices & GERD $(\mathrm{n}=36)$ & P value \\
\hline Varices (113) & $32(88.89 \%)$ & \multirow{2}{*}{$0.011^{*}$} \\
\hline No varices (37) & $4(11.11 \%)$ & \\
\hline \multicolumn{2}{|c|}{ Values expressed as number $(\%) . \mathrm{p}=0.011^{*}(\mathrm{p}<0.05)$} \\
\hline
\end{tabular}

Lower esophageal biopsies showed oesophagitis $(29.8 \%)$, and RE (36.8\%) in patients without GERD endoscopically, but patients with GERD by endoscopy (36) oe- sophageal biopsies showed Barrett s oesophagitis (22.3\%), as well as RE with mild activity $(41.6 \%)$, and RE with moderate activity $(36.1 \%)$.

Table 6: Association pathology of biopsies taken from groups

\begin{tabular}{|l|c|}
\hline Characteristics of pathology & NO GERD $(\mathrm{n}=114)$ \\
\hline Non-specific oesphagitis & $38(33.4 \%)$ \\
\hline Microscopic oesphagitis & $34(29.8 \%)$ \\
\hline reflux oesophagitis & $42(36.8 \%)$ \\
\hline Characteristics of pathology & GERD $(\mathrm{n}=36)$ \\
\hline Reflux oesophagitis with mild activity & $15(41.6 \%)$ \\
\hline Reflux oesophagitis with moderate activity & $13(36.1 \%)$ \\
\hline Barrett's oesphagus & $8(22.3 \%)$ \\
\hline
\end{tabular}

\section{Discussion}

Gastroesophageal reflux disease (GERD) is one of the commonest diseases in modern civilization affected people's health and the quality of life (Gisbert et al, 2009). GERD was a common, chronic disease that affects up to $20 \%$ of the adult population in the United States (Kahrilas, 2008), and the most common gastrointestinal diagnosis recorded among outpatient clinics (Shaheen et al, 2006). GERD contributes in excess of 10 billion $\$$ in annual direct health care costs, with the majority of cost directed to proton pump inhibitors (PPI) (Sandler et al, 2002). Guidelines issued by the American College of Gastroenterology (ACG) define GERD as symptoms or mucosal damage produced by the abnormal reflux of gastric contents into the esophagus (DeVault et al, 2005). GERD was associated with considerable morbidity and complications, such as esophageal ulcerations $(5 \%)$, peptic stricture $(4 \%-10 \%)$ and Barrett's esophagus ( $8 \%$ to $10 \%$ ), and being chronic disease significantly impairs quality of life (Enck et al, 2007).

Symptomatic GERD impairs the quality of life (QOL) of patients with chronic liver disease, thus, those patients should be assessed for the presence of symptomatic GERD, and if present, treatment should be considered in order to improve the QOL of these patients (Suzuki et al, 2008). Chronic liver disease patients, especially those with portal hypertension and liver cirrhosis, have clinical manifestations, as esophageal varices and ascites, the role of esophageal varices in developing esophageal motor disorders and abnormal gastroesophageal reflux in them (Grassi et al, 2001). Ascites proved a factor promoting gastro-oesophageal reflux, and 
questioned whether or not reflux would favor varices rupture (Navarro et al, 2003; Schechter et al, 2007).

In the present study, RE was more prevalent in males than in females $(80.5 \%$ vs. $19.5 \%$ ) with significant difference. This agreed with Li et al. (2010) who found that $\mathrm{RE}$ was more in males than females. A more progressive clinical course and a predominance of cirrhosis with comorbid related factors contributing to RE like esophageal varices and ascites by $\mathrm{Yu}$ et al. (2001) showed its' commonest in males. But, Kotzan et al. (2001) found no correlation between sex and RE.

In the present study, RE was more prevalent in the older patients with CLD (61\%) above 60 years old. This agreed with $\mathrm{Li}$ et al. (2010) who found a relationship between high RE prevalence among patients with chronic liver disease and ages. This agreed with Collen et al. (1995) and Huang et al. (1999) who found more severe gastroesophageal reflux and esophageal lesions in elderly patients as compared to younger ones. The abnormalities that play a pathogenic role in GERD were more severe in the elderly patients and lead to the increased rate of GERD complications. Also, multiple medications more frequently taken by the elderly for co-morbid illnesses such as NSAIDs, beta blockers and antidepressants, are well known to decrease LES pressure. Also many diseases that can negatively affect esophageal motility appear with greater frequency with advanced age.

In the present study, 81 patients (54\%) with GERD, 73 (48.2\%) of them had typical symptoms including heartburn, acid regurgitation and excessive salivation, but eight (5.33\%) complained of atypical symptoms. In the present study, frequency of symptomatic GERD was more prevalent than that done by Zhang et al. (2011) who reported $(32.05 \%)$ typical symptoms of gastro-esophageal reflux disease patients. They studied only 78 patients with liver cirrhosis without esophageal varices, but in the present study the majority of patients had esophageal varices demonstrated as a positive mechanical factor contributing to GERD. Also, the present results were more than that of Suzuki et al. (2008) who found that $33.6 \%$ patients with chronic liver disease had symptomatic GERD higher in chronic liver disease patients than in general population.

In the present study, heartburn was the most predominant symptom in all symptomatic patients but without significant difference between GERD symptoms and GERD different grades $(\mathrm{P}>0.05)$, denoting no relationship between GERD symptoms and disease severity. It was not possible to preview the endoscopic findings by symptoms intensity or frequency. This agreed with DeVault et al. (2005) who reported that heartburn was the classical GERD symptom. Heartburn is caused by acid stimulation of sensory nerve endings in deeper layers of the esophageal epithelium. Schechter et al. (2007) reported that the typical symptoms, GERD should be suspected, but, a correlation between presence or absence of symptoms and the reflux intensity could not be found.

In the present study, when GERD patients were endoscopically assessed according to Los Angeles classification 36/81 (44.44\%) of the symptomatic patients and $(24 \%)$ of all patients with chronic liver disease. GERD grade (B) was the most frequent grade presented in $16(44.44 \%)$ of GERD patients. This agreed with Zhang et al. (2011) who studied GERD in cirrhotic patients depending on the upper endoscopy, esophageal manometry, and 24-h esophageal $\mathrm{pH}$ monitoring. They reported a high incidence of RE (endoscopically) and pathologic reflux (by $\mathrm{pH}$ metry) in patients with severe chronic liver disease. Li et al. (2010) found significantly high prevalence of the RE $36.4 \%$ (469/1280) in patients with chronic liver disease, which was. This difference between ( $24 \%$ vs. $36.4 \%$ ) may be due to the large number of patients, which majority had ascites and esophageal varices, con- 
tributing to a higher GERD incidence. On the other hand, Ahmed et al. (1993) studied GERD in 25 cirrhotic by $\mathrm{pH}$ manometry and upper endoscopy, found a low prevalence in GERD patients with CLD and $12 \%$ of them had endoscopic evidence of esophagitis. The liver cirrhosis proved an important predispoing factor for gastroesophageal reflux, not only the mechanical effects caused by esophageal varices or ascites, but also neural and humoral factors were related to the high incidence of GERD in liver cirrhosis patients. Cárdenas et al. (2001) reported nitrous oxide (NO) in large amounts in the systemic circulation of cirrhotic patients, none showed decrease the amplitude of distal esophageal peristaltic waves, and the peristaltic contractions velocity in the proximal esophagus attributed to high incidence of GERD in liver cirrhosis patients. On the other hand, in 45/81 patients with GERD symptoms endoscopically had no RE, which was referred as non-erosive reflux disease (NERD). This agreed with Schechter et al. (2007) who studied the GERD prevalence in cirrhotic patients with esophageal varices without endoscopic treatment, and found no abnormal reflux 14/27 (52\%) patients with typical reflux symptoms. A high percentage of NERD was reported by Zagari et al. (2008) who performed a large epidemiologic study and found that $23.7 \%$ of 1,033 patients had reflux symptoms at least twice a week, of those patients with reflux symptoms, $75.9 \%$ gave a negative endoscopy.

Ronkainen et al. (2005) among 1000 northern Europeans found that two thirds of them with reflux symptoms had no esophagitis and there was imperfect correspondence between GERD symptoms and endoscopic features of the disease. All studies on patients with GERD-related symptoms suggested that the prevalence of NERD between $50 \% \& 70 \%$ due to the widespread use of proton pump inhibitors (PPI), but some studies stated that the NERD prevalence was over estimated by including healed erosive esophagitis among NERD patients. The pre- sent study showed highly significant difference $(\mathrm{P}<0.001)$ between the ascites degrees and GERD grades, as the GERD degree was higher in patients with marked ascites, who had more frequently GERD grade (C). These results agreed with Li et al. (2010) who found a significant relationship between ascites and reflux esophagitis. Ascites increased intra-abdominal pressure, compressing the stomach and its contents, and thus alter the anatomic anti-reflux elements naturally occurring against reflux, and delayed gastric half-emptying of liquid food in patients with liver cirrhosis and ascites. Bhatia et al. (1999) studied the effect of tense ascites on the esophageal body motility and lower esophageal sphincter pressure and found that esophageal body contraction wave duration increased in ascites, and decreased after its control, but the LES pressure was not affected by ascites. Navarro et al. (2003) reported that trend of reduced GERD when intra-abdominal pressure was reduced by paracentesis. They concluded that, although a significant reduction of intra-abdominal pressure occurred in controlled ascites volume, it did not correspond to any alteration in the LES pressure or LES length or LES abdominal length. However, Iman et al. (2009) in Egypt concluded that the esophageal motility and the pressure of LES in patients with and without ascites had no significant difference, which agreed with Avgerinos et al. (2002).

In the present study, there was a highly significant relationship $(\mathrm{P}<0.001)$ between the reflux esophagitis and severity of chronic liver disease graded by Child-TurcottePugh scoring system. But, without significant correlation $(\mathrm{P}=0.206)$ between GERD grades and liver disease severity. In advanced stages of liver diseases child (B) and (C) patients, GERD was more prevalent than child (A) patients. This could be attributed to the ascites and esophageal varices that are frequent findings in decompensated liver cirrhosis. This agreed with Li et al. (2010) who found a positive relationship between 
the severity of liver damage and RE and the highest prevalence of RE existed between the patients with liver failure or Child B \& C liver cirrhosis. Also, this data agreed with Zhang et al. (2011) who stated that the more liver damage severity, the more abnormal parameters of acid and bilirubin reflux. Also, they found that the reflux incidence was higher in Child $\mathrm{B}$ or $\mathrm{C}$ group than in Child A group. A stepwise increased of mixed reflux was reported along with the severity of liver function damage.

The present study showed a significant relationship $(\mathrm{P}=0.011)$ between the presence of esophageal varices and GERD. Ahmed et al. (1993) who studied by pH-metry 25 cirrhotic patients and 30 GERD patients without liver disease, and found that among the cirrhotic patients with GERD $81 \%$ had EV, and that GERD was common in cirrhotics with EV. Moreover, Schechter et al. (2007) and Zhang et al. (2011) who found that EV itself, independent of the cirrhosis, delayed the esophageal clearance and increased contact time between acid and mucosa.

Passaretti et al. (1989) and Iwakiri et al. (1993) found that motor disorders in esophageal body delayed in esophageal clearance time and abnormal gastroesophageal reflux occurred in cirrhotic patients with EV. The present study showed no significant relationship between the size of esophageal varices and GERD. This agreed with Schechter et al. (2007) who found that esophageal dysmotility occurred in the presence of varices due to the mechanical effect of the blood with in the varices, irrespective of their size and also Li et al. (2010) who found no significant relationship between EV size and RE. Also, Ahmed et al. (1993) found that GERD was common in the cirrhotic with $\mathrm{EV}$, independent of their caliber. But, when GERD was assessed by Iman et al. (2009) using esophageal manometery, they found that patients with high-grade esophageal varices had significant decrease in esophageal body amplitude in middle and distal esophagus, and explained that by the high mechanical effect of large varices, which diminished the amplitude and duration of the peristaltic waves and predisposed to GERD.

The present study showed a significant relationship $(\mathrm{P}<0.001)$ between the GERD and the presence of signs of recent variceal bleeding. This agreed with Lodato et al. (2008) who found high-grade varices and red color (RC) signs that tended to be more frequently on the right posterior wall of esophagus rather than the other areas, but bleeding varices with $\mathrm{RC}$ signs were more frequently found in the right anterior wall. Lodato et al. (2008) found that the mucosal breaks including erosions and ulcers in reflux esophagitis were most frequent on the right anterior wall of the lower esophageal mucosa, due to longer acid contact time of the lower esophagus of right anterior wall.

The longer acid contact time on the right anterior wall may damage the esophageal mucosa and increase the risk of variceal rupture, and aided by the delayed acid clearance in patients with liver cirrhosis and comorbid factors lower esophageal biopsies were taken and were pathologically examined, some patients who were negative endoscopically, had GERD pathologically and one of those patients was having esophagitis with mild dysplasia. Also Zagari et al. (2008) reported that a large number of GERD patients were negative at upper endoscopy.

Lundell et al. (2009) stated that the histological characteristics diagnosed GERD mainly the NERD. NERD patients had the highest number of acid reflux events before sensed reflux event, which suggested that prior sensitization was needed for an acid reflux to be perceived in NERD patients with a lower acid exposure compared with erosive esophagitis patients. The distal amplitude contractions, as well as mean lower esophageal resting pressure, are mildly reduced in NERD patients in contrast to those with the erosive esophagitis who demonstrate obvious peristaltic dysfunction. 


\section{Conclusion}

Generally speaking, the esophagitis (or oesophagitis) is an esophagus inflammations. It may be acute or chronic. The acute esophagitis can be the catarrhal or phlegmonous, whereas chronic esophagitis may be hypertrophic or atrophic.

The outcome data showed that the high prevalence of RE (24\%) was demonstrated among upper endoscopies in Egyptian patients with liver cirrhosis presented to $\mathrm{Al}$ Azhar university hospital with overall $54 \%$ of the studied patients were complaining of the classical GERD symptoms. Heartburn is the classical symptom of GERD, but was not an indicator for the disease severity, and was not possible to preview the endoscopic and microscopic findings by the intensity or frequency of symptoms. On one hand, a significant relationship was demonstrated between the GERD, esophageal varices, and ascites, and signs of the recent bleeding on the other hand.

\section{References}

Ahmed, AM, Al Karawi, MA, Shariq, S, Mohamed, AE, 1993: Frequency of gastroesophageal reflux in patients with liver cirrhosis. Hepatogastroenterology 40:478-80.

Avgerinos, A, Viazis, N, Armonis, A, Vlachogiannakos, J, Rekoumis, G, et al, 2002: Early increase of lower oesophageal sphincter pressure after band ligation of oesophageal varices in cirrhotics: an intriguing phenomenon. Eur. J. Gastroenterol. Hepatol. 14:1319-23.

Bhatia, SJ, Narawane, NM, Shalia, KK, Mistry, FP, Sheth, MD, et al, 1999: Effect of tense ascites on esophageal body motility and lower esophageal sphincter pressure. Indian J. Gastroenterol. 18:635-7.

Cárdenas, A, Ortega, R, Ginés, P, 2001: Hepatocirculatory syndrome in cirrhosis: Therapy in hepatology. Barcelona Arch. Med. 33-41.

Christensen, E, Schlichting, PS, Fauerhol- dt, L, Gluud, EC, Andersen, PK, et al, 1984: Prognostic value of Child-Turcotte criteria in medically treated cirrhosis. Hepatology 4:430-5.

Collen, MJ, Abdulian, JD, Chen, YK, 1995: Gastroesophageal disease in the elderly: more severe disease that requires aggressive therapy. Am. J. Gastroenterol. 90:1053-9.
Cooper, A, Gisbert, JP, Karagiannis, D, Hatlebakk, J, Agreus, L, et al, 2009: Impact of gastro esophageal reflux esophagitis on patients 'daily lives: An European observational study in the primary care setting. Hlth. Quality of Life Outcomes, 7:60

DeVault, KR, Castell, DO, 2005: Updated guidelines for the diagnosis and treatment of gastroesophageal reflux disease. Am. J. Gastroenterol. 100:190-7.

Enck, P, Dubois, D, Marquis. P, 2007: Quality of life in patients with upper gastrointestinal symptoms: results from the domestic/ international gastroenterology surveillance study (DIGEST). Scand. J. Gastroenterol. 34:48-53.

Fock, KM, Talley, NJ, Fass, R, Goh, KL, Katelaris, P, et al, 2009: Asia-pacific consensus on the management of gastro esophageal reflux esophagitis: update. J. Gastroenterol. Hepatol, 23, 3:504-8.

Garcia-Tsao, G, Jaime, B, Groszmann, RJ, 2008: Portal hypertension and variceal bleedingunresolved. Hepatology 47, 5:1764-72.

Grassi, M, Albiani, B, De Matteis, A, Fontana, M, Lucchetta, MC, et al, 2001: Prevalence of dyspepsia in liver cirrhosis: a clinical and epidemiological investigation. Minerva Med. 92:712.

Hammer, HF, 2009: Reflux-associated laryngitis and laryngopharyngeal reflux: a gastroenterologist's point of view. Dig. Dis. Sic. 27, 1: 147.

Huang, XH, Zhu, HM, Deng, CZ, Porro, GB, Sangaletti, O, et al, 1999: Gastroesophageal reflux: the features in elderly patients. World J. Gastroenterol. 5:421-3.

Iman, R, Bahaa, Y, Hesham, EM, Hafez, HA, Ragheb, M, et al, 2009: Study of oesophageal motility in Egyptian cirrhotic patients before and after prophylactic endoscopic variceal ligation. Arab J. Gastroenterol. 10:49-56.

Iwakiri, K, Kobayashi, M, Sesoko, M, Nomura, T, 1993: Gastroesophageal reflux and esophageal motility in patients with esophageal varices. J. Gastroenterol. 4:477-82.

Kahrilas, PJ, 2008: Complications of GERD. In: MD Up To Date: Nicholas, J, Talley and Peter, AL, Bonis; Deputy Editor.

Kotzan, J, Wade, W, Yu, HH, 2001: Assessing NSAID prescription use as a predisposing factor for gastroesophageal reflux disease in a Medicaid population. Pharm. Res. 18:1367-72. 
Lemme, EMO, Coelho, HSM, Schechter, RB, 2007: Gastro esophageal reflux in cirrhotic patients with esophageal varices without endoscopic treatment. Arq. Gastroenterol. 44, 2: 14550.

Li, B, Zhang, B, Ma, JW, Li, P, Li, L, et al, 2010: High prevalence of reflux esophagitis among upper endoscopies in Chinese patients with chronic liver diseases. BMC Gastroenterol. 10:54-8.

Lodato, F, Azzaroli, F, Di Girolamo, M, Feletti, V, Cecinato, P, et al, 2008: Proton pump inhibitors in cirrhosis: Tradition or evidence based practice? World J. Gastroenterol. 14, 19: 2980-5.

Lundell, LR, Dent, J, Bennett, JR, Blum, AL, Armstrong, D, et al, 2009: Endoscopic assessment of esophagitis: clinical and functional correlates and further validation of the Los Angeles classification. Gut 45, 2:172-80.

Mumtaz, K, Ahmed, US, Abid, S, Baig, N, Hamid, S, et al, 2010: Precipitating factors and the outcome of hepatic encephalopathy in liver cirrhosis. J. Coll. Physic. Surg. Pakis. 20: 514-8.

Navarro-Rodriguez, T, Hashimoto, CL, Carrilho, FJ, Strauss, E, Laudanna, AA, et al, 2003: Reduction of abdominal pressure in patients with ascites reduces gastroesophageal reflux. Dis. Esoph. 16:77-82.

Okamoto, E, Amano, Y, Fukuhara, H, Furuta, K, Miyake, T, et al, 2008: Does gastroesophageal reflux have an influence on bleeding from esophageal varices? J. Gastroenterol. 43, 10:803-8.

Passaretti, S, Mazzotti, G, deFranchis, R, Cipolla, M, Testoni, PA, et al, 1989: Esophageal motility in cirrhotics with and without esophageal varices. Scand. J. Gastroenterol. 24: 334-8.

Ronkainen, J, Aro, P, Storskrubb, T, Johansson, SE, Lind, T, et al, 2005: Prevalence of the Barrett's esophagus in the general population: an endoscopic study. Gastroenterology 129: 182531.

Sandler, RS, Everhart, JE, Donowitz, M, Adams, E, Cronin, K, et al, 2002: The burden of selected digestive diseases in the United States. Gastroenterology 122:1500-1.

Schechter, RB, Lemme, EM, Coelho, HS, 2007: Gastroesophageal reflux in cirrhotic patients with esophageal varices without endoscopic treatment. Arq Gastroenterol; 44: 145-50.

Shaheen, NJ, Hansen, RA, Morgan, DR, Gangarosa, LM, Ringel, YR, et al, 2006: The burden of gastrointestinal and liver diseases. Am. J. Gastroenterol. 101:2128-38.

Suzuki, K, Suzuki, K, Koizumi, K, Takada, H, Nishiki, R, et al, 2008: Effect of symptomatic gastro esophageal reflux disease on quality of life of patients with chronic liver disease. Hepatol. Res. 38, 4:335-9.

Yu, MW, Yang, YC, Yang, SY, Cheng, SW, Liaw, YF, et al, 2001: Hormonal markers \& hepatitis B virus-related hepatocellular carcinoma risk: a nested case-control study among men. J. Natl. Cancer Inst. 93:1644-51.

Yu, ZL, 2009: Consensus on quantitative grading of endoscopic varices. Zhong Hua Xiao Hua Nei Jing Za Zhi 17, 4:197-8.

Zagari, RM, Fuccio, L, Wallander, MA, Johansson, S, Fiocca, R, et al, 2008: Gastro-oesophageal reflux symptoms, oesophagitis and Barrett's oesophagus in the general population: the Loiano-Monghidoro study. Gut 57:1354-9. 